\title{
Measuring the T Wave of the Electrocardiogram; The How and Why
}

\author{
A. van Oosterom \\ Department Cardiomet, Faculty of Biology and Medicine; University of Lausanne, Lausanne, Switzerland; e-mail: \\ adriaan.vanoosterom@epfl.ch
}

\begin{abstract}
The genesis of the $T$ wave of the electrocardiogram is discussed on the basis of a biophysical model, derived from the underlying electrophysiology. This model is used to scrutinize the commonly-assumed significance of the individual features of this waveform. As is shown, the last word is yet to be said about this topic.
\end{abstract}

Keywords: T wave morphology; QT level abnormalities, Timing Apex, T wave duration.

\section{INTRODUCTION}

$\mathrm{T}$ HE T WAVE is one of the prominent features of the electrocardiogram (ECG). An example is presented in Fig.1, where it is shown next to the other wavelets commonly used in describing the major features of the ECG: P, Q, R, S and $\mathrm{U}$. This nomenclature was introduced by Einthoven in 1900 [1]. The labels PQRST were chosen by Einthoven for no other reason than that a labeling starting with $\mathrm{A}, \mathrm{B}$, etc. was already in use at the time for other types of components of signals related to the heart, significantly those of heart sounds. After some initial try-outs, the significance of most of the individual wavelets soon became recognized.

The QRS wavelets, elements of the QRS complex during the so-called QRS interval, are attributed to the depolarization phase of the heart's ventricles, initiating and accompanying their mechanical activity. The subsequent $\mathrm{T}$ wave signals are associated with the subsequent return of the transmembrane potentials (TMPs) of the ventricular myocytes to their resting state.

The $\mathrm{P}$ wave, preceding the QRS complex, is generated by the electric activity of the atria that supports the filling of the ventricular cavities prior to the contraction of the ventricular walls. Curiously, the significance of the $U$ wave is currently, more than a century after Einthoven's work, still the subject of debate $[2,3]$.

During the twentieth century, the ECG developed into one of the most important non-invasive tools of electrocardiology [4]. Contributions came from fields such as basic electrophysiology, clinical cardiology, biophysical modelling, improved recording technology and, during the final decades, the field of signal processing supported by computer science.

The widespread application of the ECG has brought about an ever-growing demand on the diagnostic accuracy of the technique. Again, curiously enough, more than a century later, its full potential has not yet been reached, and several basic problems still remain to be resolved.

In the diagnostic application of the ECG, two different categories can be distinguished: the analysis of heart rhythm and that of the waveforms observed in the ECG. This invited, didactic paper relates to the latter problem, and in particular to the significance of the features observed in $\mathrm{T}$ wave morphology.

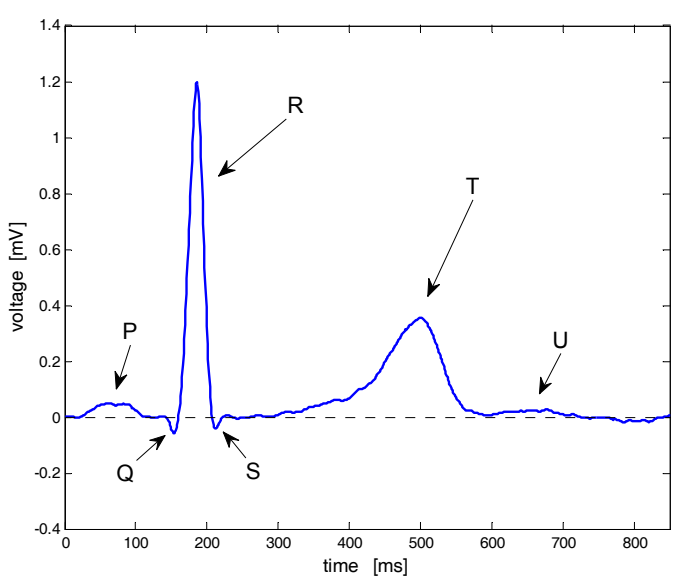

Fig.1 Nomenclature of wavelets of the ECG as recorded on a healthy subject (lead V4)

\section{MEASURING THE T WAVE; HOW?}

These days, the technology for measuring bio-electric signals has attained a high quality $[5,6]$. The basic principle involved is straightforward. All it amounts to is the placing of two electrodes at different locations on the thorax, which sense the potential difference between these two locations; in the case of the ECG resulting in a signal generated by electric current sources inside the myocardium.

The potential differences on the body surface are transferred to the amplifier by placing electrodes on the skin. At the interface between the skin and the -commonly usedmetal electrodes an exchange of charge takes place between the electrons inside the metal and ions inside the body tissues. As a consequence, voltage differences build up at both interfaces, having magnitudes that may be much greater than those of the ECG itself. Moreover, the polarity and magnitude may change slowly over time in response to changes in skin temperature and moisture, as well as any pressure exerted. Significantly, these changes are unequal at the two interfaces, with the differences in the so-called half-cell potentials feeding directly into the amplifier. To reduce the effect of such artefacts, the DC type of components in the ECG, components having frequencies below $0.1 \mathrm{~Hz}$, are invariably suppressed by 
analogue or digital filtering in the first stages of the recording system. A direct consequence of this is that in the signals available for diagnostic purposes, the DC component, being the mean value of the signal over a single beat, is irreparably lost. This is highly relevant, in particular for the interpretation of the ECG during the QT interval, as is evident later on in this paper.

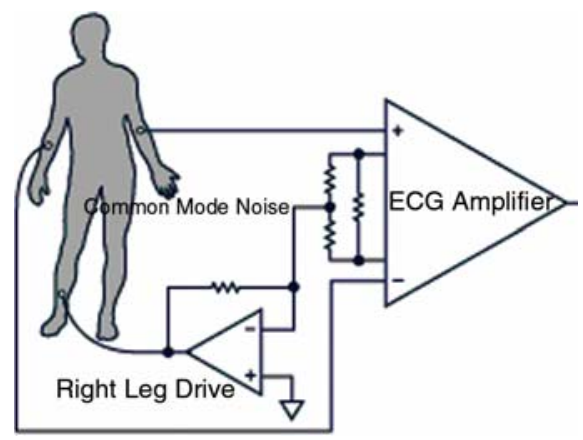

Fig.2 Basic principle used for recording a single ECG, sensing the potential difference generated by the heart's electric activity between the left and the right arm. The third electrode shown, the one on the right leg, serves the very important purpose of minimizing the contribution of electromagnetic fields external to the body on the observed signal [7].

\section{Measuring the T Wave; But Which one?}

Measuring bioelectric potentials requires the use of at least two electrodes since the physics involved indicates that electric potential as such is specified uniquely up to a constant only, and only potential differences between different locations are those that signify.

In ECG terminology, the signal observed between two terminals of any electrode configuration is referred to as a "lead". The configuration shown in Fig.2 relates to the recording of a single lead. The third electrode serves to suppress any interferences stemming from electromagnetic fields in the environment, in particular when using a mainspowered amplifier. This third electrode does not, or at least should not, influence the waveform of the recorded signal. Due to this, such electrodes do not play a role in the subsequent discussion.

\section{A. Multilead signals}

In most applications the ECG is recorded by placing more than two electrodes on the thorax. The most frequently used electrode montage is that of the standard 12-lead system, depicted in Fig.3. Note that this only involves 9 electrodes. The thorax is that of a 54-year-old healthy male (subject HM), a numerical model based on his magnetic resonance (MR) images. It includes ventricles, atria and lungs, as used in the computation of the effects of the shape and composition of the volume conductor (the body tissues) on the observed ECG waveforms. The lead signals for a single beat observed at these locations are shown in Fig.4.
As can be seen, the 12-lead system involves nine electrodes only. The signals observed over the time interval of a single beat are shown in Fig.4. The columns 2, 3 and 4 represent the signals observed at the electrode locations shown in Fig.3 acting as one of the terminals of the lead signals -usually referred to as sensing terminals-, the other terminal being the mean of the potentials present at electrodes VR, VL and VL, the so-called Wilson Central Terminal (WCT), acting as the reference terminal. This distinction is relevant only in cases where multiple signals are recorded with reference to a single, common terminal.

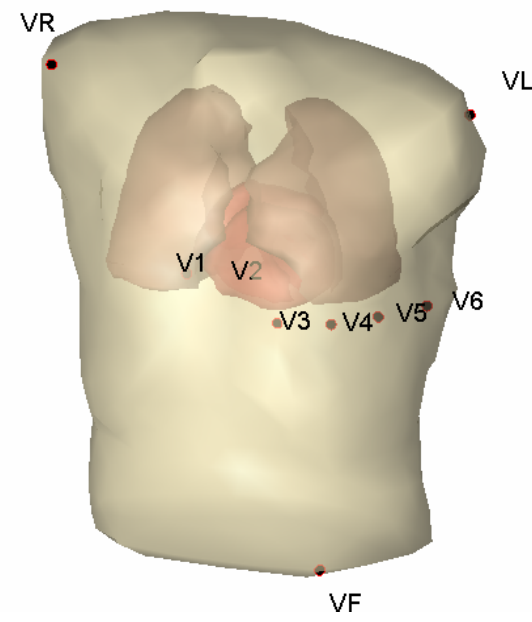

Fig.3 Electrode montage of the standard 12-lead system. Note that this only involves 9 electrodes. The geometry depicted is that of the subject's thorax based on his magnetic resonance (MR) images.

When referenced to WCT (but only then!), the sum of the potentials of leads, VR, VL and VF, is zero and, hence, at most eight independent signal components are contained in the signals of the standard 12-lead system.

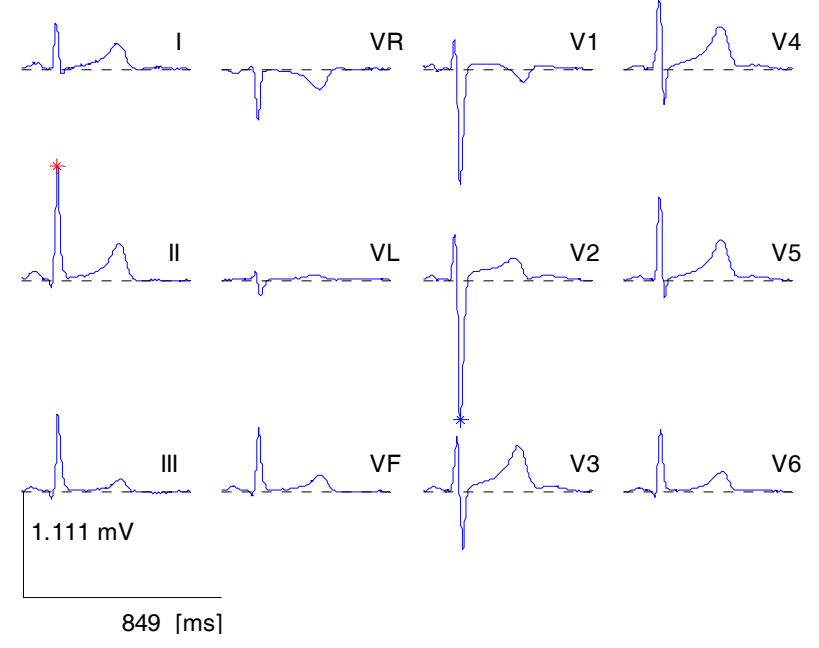

Fig.4 ECG waveforms of subject HM recorded by means of the standard 12-lead system during one complete heart beat; electrode positions as shown in Fig.3. 
The signals in the first column of Fig. 4 are the three linear combinations that can be formed on the basis of the potentials at VR, VL and VF, e.g. II=VF-VR. As such these so-called bipolar leads (a misnomer: all recorded signals involve two terminals, electrodes, or "poles") do not carry extra information. The redundancy involved in the 12 lead signals has proved to be valuable for diagnostic applications of the ECG on the basis of visual inspection of its waveforms. Various other electrode montages are used in specific applications, involving either fewer or more electrode locations for sampling the spatial aspect of the electric field on the thorax [8-10].

\section{B. Signal features}

The ECGs of normal subjects, the ECG waveforms of individual leads exhibit clear inter-individual differences. In a hybrid type of convention, the nomenclature of the Q, R and S wavelets of the QRS complex are strictly coupled to their polarity; early negativity: negative $S$, subsequent positivity: $R$ and subsequent negativity: $\mathrm{S}$. In contrast, the polarity of the other wavelets: $\mathrm{P}, \mathrm{T}$ and $\mathrm{U}$ may be either positive or negative and biphasic variants can also be observed. In the example shown (Fig.4), it can be seen that in an individual lead, not all of these wavelets may be present.

Waveform features used in diagnostic applications of the ECG are timing, magnitude and duration of the individual wavelets. For each of these, in all of the leads of the standard 12-lead system, extensive sets of normal values have been collected [11]. Unfortunately, all these normal values were found to have a large standard deviation, which hampers the classification of distinct types of cardiac disease on the basis of such features. Even when using multivariate types of analysis and applying stratifications on the basis of gender, age or other constitutional variables, there remains a clear need to improve the diagnostic performance. In particular, for the $T$ wave, no indication is given about the lead on which the $\mathrm{T}$ wave should preferably be analyzed.

\section{MEASURING THE T WAVE; WHY?}

The statistical analysis of large ECG data bases, in combination with clinical observations, has revealed that the $\mathrm{T}$ wave, and in particular the signals during the interval leading up to its apex, the ST segment, contains useful diagnostic information, in particular with respect to the episodes of acute ischemia that might lead to myocardial infarction (MI). In extreme cases, when effecting particular regions of the ventricles, the departures from the normal range are indicative. Other interest in $\mathrm{T}$ wave morphology stems from the more recently identified hazards arising from repolarization abnormalities, expressed in the more extreme departures of the waveform normality in the variants of the Long-QT syndrome [12], the Brugada syndrome [13] and the Short-QT syndrome [14]. All of these syndromes are associated with lifethreatening heart rhythm disturbances.
In all of the above-mentioned cases, strict diagnostic criteria that can be applied to the ECG are either lacking or of insufficient quality. Widely differing opinions about the optimal features of the $\mathrm{T}$ wave to be used, and their significance in terms of the underlying electrophysiology can be seen in the literature. Improvement will probably be slow if derived from statistical analysis only; however, the incidence of these syndromes is, fortunately, low. Another approach is to try to understand the involved basic mechanisms, research that is currently being carried out in many research centres. This concerns in particular work at the cellular and membrane level, at which an impressive collection of distinct, genetically related defects have been identified.

On a larger scale, the link between cellular activity during repolarization and the resulting ECG waveforms, much less research activity is seen to be carried out. It is here that the biophysical modelling of the genesis of the $\mathrm{T}$ wave comes into play, an approach that may lead to the improved use of $\mathrm{T}$ wave features and the understanding of their limitations.

Without being always aware of the underlying model-based assumptions, invariably all measurements taken, and in particular the interpretations of their numerical values, are linked to a model [15]. This applies equally to the measuring of the $\mathrm{T}$ wave and its diagnostic interpretation.

\section{Modeling The T WAVE; HOW?}

\section{A. Theory}

The electrocardiogram arises from the electric activity at the membranes of the cardio-myocytes, the contractile elements of the heart muscle. In the following, the myocardium is modelled as an ensemble of myocytes, with their intracellular domains coupled by sets of intercalated discs. On a macroscopic scale, the membrane processes at a location $\vec{r}$ at time instant $t$ feed an electric current into the extracellular domain, with source surface density $\vec{J}^{\mathrm{i}}(\vec{r}, t)$ (unit: $\mathrm{A} / \mathrm{m}^{2}$ ). As implied in the work of Wilson [16], and later derived explicitly by Plonsey [17], the impressed density is related to the local transmembrane potential by

$$
\vec{J}_{\mathrm{m}}(\vec{r}, t)=\nabla\left(\sigma_{\mathrm{i}} V_{\mathrm{m}}(\vec{r}, t)\right)
$$

in which $\nabla$ denotes the gradient operator (unit:1/m), $\sigma_{\mathrm{i}}$ the electric conductivity of the intracellular domain (unit: $\mathrm{S} / \mathrm{m}$ ), and $V_{\mathrm{m}}$ is the local transmembrane potential (unit: $\mathrm{V}$ ).

In the passive extracellular domain, based on the conservation of charge, the impressed current density $\vec{J}^{\mathrm{i}}(\vec{r}, t)$ sets up an electric field $\vec{E}(\vec{r}, t)$ (unit: $\mathrm{V} / \mathrm{m}$ ) (a vector field) that should satisfy the differential equation

$$
\nabla \cdot \sigma_{\mathrm{e}} \vec{E}(\vec{r}, t)=-\nabla \cdot \vec{J}_{\mathrm{m}}(\vec{r}, t)
$$

in which $\nabla$. denotes the divergence operator, (unit: $1 / \mathrm{m}$ ), and $\sigma_{\mathrm{e}}$ the electric conductivity of the external medium. As one of the basic results of potential theory, we find that, in an 
external medium of infinite extent, the electric field $\vec{E}(\vec{r}, t)$ is the negative gradient of an "auxiliary", scalar function $\Phi\left(\vec{r}^{\prime}, t\right)$, called the electric potential at field point $\vec{r}^{\prime}$. The potential reference involved is conveniently placed at infinity, where the potential is defined as zero. The potential is found by integration over all of space, justified by the superposition principle that applies to a linear medium:

$$
\Phi\left(\vec{r}^{\prime}, t\right)=-\frac{1}{4 \pi \sigma_{\mathrm{e}}} \int \frac{\nabla \cdot \vec{J}_{\mathrm{m}}(\vec{r}, t)}{R} \mathrm{~d} V
$$

in which $R$ denotes the length of the vector $\vec{R}=\vec{r}^{\prime}-\vec{r}$ pointing from source location $\vec{r}$ to observation point (field location) $\vec{r}^{\prime}$.

Next, by combining (1) and (3) we have

$$
\Phi\left(\vec{r}^{\prime}, t\right)=-\frac{1}{4 \pi \sigma_{\mathrm{e}}} \int_{\mathrm{V}} \frac{\nabla \cdot \nabla\left(\sigma_{\mathrm{i}} V_{\mathrm{m}}(\vec{r}, t)\right)}{R} \mathrm{~d} V
$$

In the final step we recall that we are dealing with cardiac sources located within a volume $\mathrm{V}$ (the myocardium), bounded by a closed surface $S$ (bounded by the endocardium and epicardium) and then apply Green's second theorem from potential theory [18] (dating from 1828) to the integral in (4), for field points outside the myocardium. Moreover, we take $\sigma_{\mathrm{i}}$ to be a uniform scalar function within the source region. This permits $\sigma_{\mathrm{i}}$ to be moved to the front of the integral. Green's second theorem applied to the scalar fields $V_{\mathrm{m}}$ and $1 / R$ reads:

$$
\int_{\mathrm{V}} \frac{1}{R} \nabla^{2} V_{\mathrm{m}} \mathrm{d} V-\int_{\mathrm{V}} V_{\mathrm{m}} \nabla^{2} \frac{1}{R} \mathrm{~d} V=\int_{\mathrm{S}} \frac{1}{R} \nabla V_{\mathrm{m}} \cdot \mathrm{d} \vec{S}-\int_{\mathrm{S}} V_{\mathrm{m}} \nabla \frac{1}{R} \cdot \mathrm{d} \vec{S}
$$

with $\mathrm{d} \vec{S}$ denoting a small element of the surface $\mathrm{S}$, a vector directed along the outward normal of S. The integral to the left of the equality sign is zero, since $\nabla^{2} \frac{1}{R}=0$ for field points outside $S$ [19]. The integral to the right of the equality sign is clearly zero since we take $\mathrm{S}$ to be as close as possible to the myocardium while keeping free of it. In the remaining integral on the right, the term

$\nabla^{2} \frac{1}{R} \cdot \mathrm{d} \vec{S}$ is, $\mathrm{d} \omega=\mathrm{d} \omega\left(\vec{r}^{\prime}, \vec{r}\right), \quad$ a scalar function signifying the solid angle subtended by an infinitesimally small element of S as viewed from the observation point. After combining these results with (4), we have

$$
\Phi\left(\vec{r}^{\prime}, t\right)=-\frac{\sigma_{\mathrm{i}}}{4 \pi \sigma_{\mathrm{e}}} \int_{S} V_{\mathrm{m}}(\vec{r}, t) \mathrm{d} \omega
$$

This expression shows that, under the assumed condition of a homogeneous intracellular conductivity, the potential in the region exterior to the active sources, i.e. outside the myocardium, depends exclusively on the transmembrane potentials at the surface bounding it. The term $\sigma_{\mathrm{i}} V_{\mathrm{m}}$ expresses the strength of an equivalent current dipole layer surface density (unit: $\mathrm{Am} / \mathrm{m}^{2}$ ). Multiplied by the surface element $\mathrm{d} \vec{S}$, a vector directed along the outward normal of $\mathrm{S}, \sigma_{\mathrm{i}} V_{\mathrm{m}}$ takes on the nature of a current dipole (unit: Am). It represents a double layer of electric current sources and sinks, and is, accordingly, referred to as an equivalent double layer (EDL), the instantaneous strength of which is proportional to $V_{\mathrm{m}}$.

This highly significant result was first described by Geselowitz in 1989 [20]. As shown, it follows directly from the classic potential theory. In Chapter 7 of the second edition of Comprehensive Electrocardiology [21], it is shown that it forms a direct extension of the application of the solid angle theory, originally used for the modelling of the depolarization phase of the myocytes (QRS complex), to that of the subsequent repolarization phase. In addition, it discusses various implications of the situations in which the assumed uniformity and isotropy of the electric conductivity of the myocardium cannot be taken to be realistic (see also [22]) as well as its relationship with the more general bi-domain theory.

\section{B. Numerical Implementation}

The use of (6) for simulating body surface potentials requires the specification of the surface $\mathrm{S}$ and of the distribution of the transmembrane voltage $V_{\mathrm{m}}(\vec{r}, t)$. A numerical variant of (6) reads

$$
\boldsymbol{\Phi}=\boldsymbol{\Omega} \mathbf{V}_{\mathrm{m}}
$$

expressing the multiplication of a matrix $\mathbf{V}_{\mathrm{m}}$ of transmembrane potentials at $T$ discrete time instants on a dense grid of $N$ nodes on the ventricular surface $\mathrm{S}$ (matrix size $N \times T$ ) by matrix $\boldsymbol{\Omega}$ (matrix size $K \times N$ ), the elements of which are (scaled) variants of the solid angles subtended by the sources around the $N$ nodes and $K$ observation points in the infinite homogeneous medium. This yields matrix $\boldsymbol{\Phi}$ (matrix size $K \times T$ ) expressing the potentials at the corresponding time instants.

In the period preceding the onset of ventricular electric activity the observed potential (differences) at the thorax surface is minimal, and generally assumed to be zero. During this period the transmembrane potential of all myocytes is taken to be at their resting potential level (about $-80 \mathrm{mV}$ ). Because of this, matrix $\boldsymbol{\Omega}$ must have a unit eigenvalue, with corresponding unit vector $\mathbf{e}$ :

$$
\boldsymbol{\Omega e}=\mathbf{o}
$$

This agrees with the well-known property of a closed surface: it subtends a zero solid angle at an exterior observation point. This, in turn, corresponds to the well-known fact that a completely uniformly polarized cell does not generate an external electric field [16].

In practical implementations of this source description, the cardiac sources are obviously not situated in an infinite medium, but rather in a bounded medium: no electric current passes normally through the skin into the non-conductive 
external medium: air. The effect of this major inhomogeneity can be accounted for by the application of the boundary element method (BEM) [23]. This method generates a linear transfer function between the potentials at all interfaces while assuming the medium to be infinite and homogeneous, and the potentials in an inhomogeneous, bounded state at the same locations [24]. In this way, major inhomogeneities, like the one at the thorax surface and those resulting from the low electric conductivity of lung tissue and the high conductivity of blood inside the ventricles, can be taken into account. These are the compartments of the numerical thorax model illustrated in Fig.3. Its numerical expression is that of a matrix transfer matrix B (matrix size $L \times K$ ), with $L$ the number of electrode locations on the thorax.

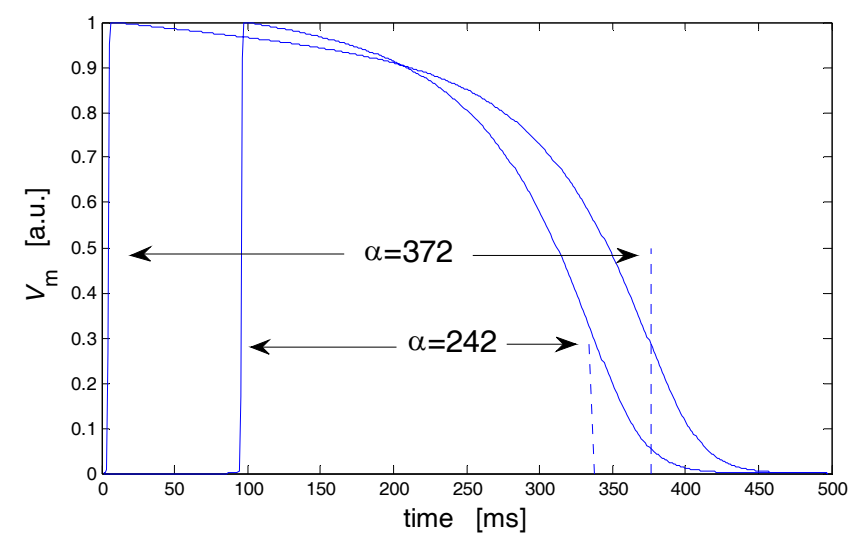

Fig.5 Examples of two waveforms modelling the trans - membrane potential $\mathrm{V}_{\mathrm{m}}(t)$ as a product of three logistic functions. The intervals between the arrows denote the activation recovery intervals.

The final, general expression for finding the simulated potentials $\mathbf{V}_{\mathrm{b}}$ in the bounded, inhomogeneous medium then reads [25]

$$
\mathbf{V}_{\mathrm{b}}=\mathbf{B} \boldsymbol{\Omega} \mathbf{V}_{\mathrm{m}}=\mathbf{A} \mathbf{V}_{\mathrm{m}}
$$

with matrix $\mathbf{A}$ expressing the general transfer between the transmembrane potentials on $\mathrm{S}$ and the potentials on the body surface. Note that $\mathbf{A e}=\mathbf{0}$, a property "inherited" from (8). Moreover, since the conductive medium involved (the bounded thorax) no longer permits defining the reference for the potential at infinity, a reference now has to be defined. The WCT reference is the one used by convention.

The effectiveness of the EDL source model has been demonstrated in several publications, some related to the forward problem as such: the simulation of the potentials based on assumed EDL source parameters [26, 27], others to inverse application: the computation of its parameters on the basis of observed body surface potentials [28-30]. This source model is the one used in ECGSIM [31], an interactive computer program for simulating QRST waveforms, downloadable, free of charge, from www.ecgsim.org.

\section{EDL-BASED MODELING OF THE T WAVE.}

We now look at some examples of $T$ waves, modeled on the basis of the EDL source model. As indicated above this demands the specification of individual transmembrane potentials $V_{\mathrm{m}}$ at all of a dense set of $N$ nodes evenly distributed over the ventricular surface $\mathrm{S}$. In the examples shown these in turn are specified by products of a number of logistic functions (hyperbolic tangents), functions of the type

$$
\frac{1}{1+e^{-\beta(t-\tau)}}
$$

These sigmoid functions having a maximum slope $\beta / 4$ at $t=\tau_{n} n=1 \cdots N$, (note: individual timing values $\tau_{n}$ for each node) [32]. As is seen in the examples of this function shown in Fig.5, three such functions are involved, one for modeling the upstroke of the transmembrane potential $V_{\mathrm{m}}$, the other two for modeling its down slope: an initial slow phase, phase 2 of the transmembrane potential, followed by its much faster phase 3 . For each individual $V_{\mathrm{m}}$, just two parameters are used, $\tau=\delta$ setting the timing of local depolarization, and $\tau=\rho$ specifying the timing of the inflection point of the downslope, taken as a marker for local repolarization. The shape parameters $\beta$ are taken to be identical for all. The parameter shown in Fig. 5 is the activation recovery interval: $\alpha=\rho-\delta$, an indicator of the local absolute refractory period of the local myocytes [33]. Note that, for uniform values of $\beta$, the shape of the TMP wave forms are a function of $\alpha$ only. The transmembrane potential is specified in arbitrary units. Its zero level is irrelevant because of (8), its magnitude is incorporated in the transfer matrix $\mathbf{A}$.

\section{B. The normal T wave}

The ECG waveforms of a healthy subject were simulated on the basis of the EDL source model. The values of local depolarization $\delta_{n}$ and repolarization $\rho_{n}$ at each of 317 nodes on $\mathrm{S}$ were derived from a dedicated inverse procedure, involving the thorax model shown in Fig. 3 and ECG signals of a 64-lead system involving 65 electrodes "strategically" placed on the thorax. This procedure is similar to the one used for activation imaging published in [28], now extended in order to include the repolarization phase. The $\delta_{n}$ and $\rho_{n}$ values, mapped on the ventricular surface $\mathrm{S}$ are shown in Fig.6a. The waveforms of the transmembrane potential $V_{\mathrm{m}}$ were of the type specified in the previous sub-section. The validation of these kinds of results is impossible. The only way to estimate its realism is by comparing the sequences with what is known from invasive electrophysiology. For the depolarization sequence, the left part of Fig.6a, the outcome is in full qualitative agreement with the unique data set published by Durrer and his colleagues in Amsterdam [34]. For the repolarization sequence, shown in Fig.6b, no such data are 
available, which is in fact the reason for the ongoing discussion on this topic mentioned in the introduction. However, the results were in agreement with those from the sparse invasive studies available $[35,36]$. Moreover, the results are in full qualitative agreement with those observed, by means of essentially the same method, when applied to several healthy individuals. The ECGs simulated on the basis of the activation and recovery sequences depicted in Figs. 6a,b are represented in Fig.7, superimposed on the corresponding measured data.

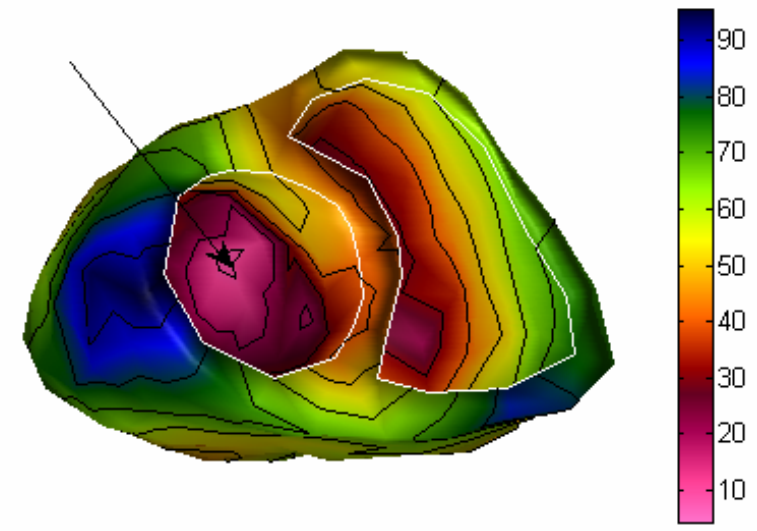

Fig.6a Posterior view of the ventricles in their natural orientation. Isochrones, drawn at $10 \mathrm{~ms}$ intervals, specify the timing of depolarization $\delta$. The arrow shown indicates the earliest activated region at the septum separating left and right ventricle. The white contours mark the transition between the endocardial and epicardial aspect of the ventricles.

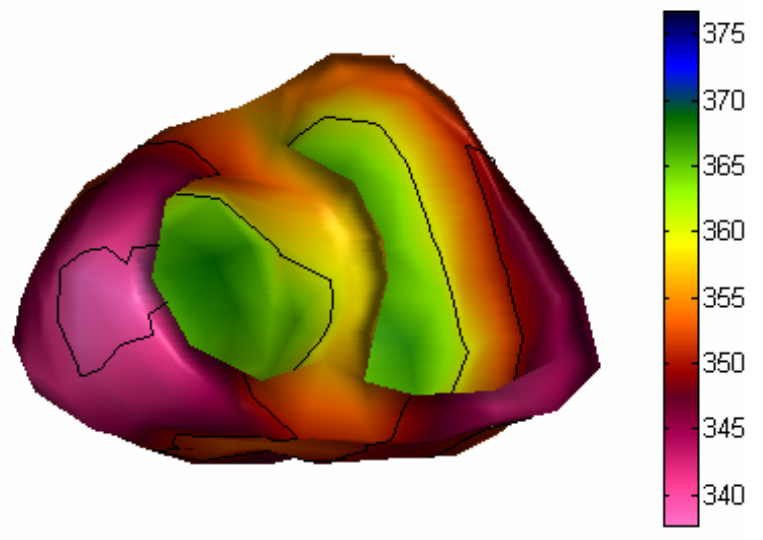

Fig.6b Posterior view of the ventricles in their natural orientation. Isochrones, drawn at $10 \mathrm{~ms}$ intervals, specify the timing of repolarization $\rho$. Note the small range of repolarization values relative to those of depolarization and its overall shift in time. The sequence of repolarization is largely, but never entirely, the reverse of that of depolarization.

\section{EDL-BASED ANALYSIS OF NORMAL T WAVE MORPHOLOGY}

The EDL source model is now discussed in its application to ascertaining the significance of major $\mathrm{T}$ wave morphology and of ST segment changes during periods of acute ischemia.

\section{A. Timing of the T wave}

The single parameter model for the timing of repolarization, $\rho$ permits a study of its effect on basic T wave morphology. While $\rho$ is a single parameter, in the EDL model it is specified for all of the $N$ small elements of the surface carrying the source, $\rho_{n}$. Even if the distribution of $\rho_{n}$, like the one shown in the Fig.6b, does not represent the full reality, its subsequent, global variation provides an interesting insight into the involved mechanisms, as is illustrated in the subsequent examples.

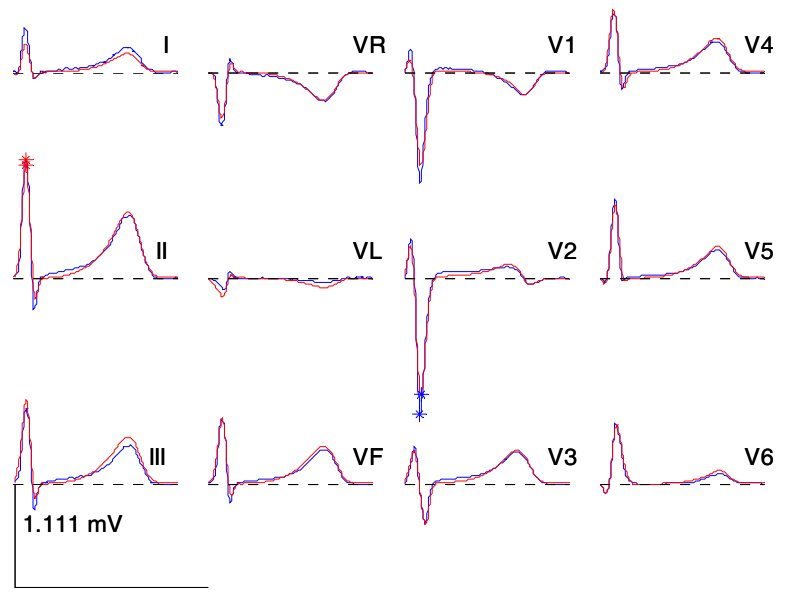

Fig.7 Superposition of the standard 12-lead signals of subject HM and those simulated on the basis of the EDL source model.

The non-uniformity of the timing of repolarization is commonly referred to as its dispersion, both for the entire myocardium as well as for transmural (endo-epicardial) differences. Based on an available set of $\rho_{n}$ values it can be quantified more precisely. Its full significance can only be appreciated by mapping it over the entire ventricular surface. A more crude documentation of dispersion is to state its basic parameters of descriptive statistics, such as the mean value $\bar{\rho}$, standard deviation $\tilde{\rho}$ and range $\vec{\rho}$.

For the distribution of $\rho$ shown in the left panel of Fig.6, these values were: $\bar{\rho}=355 \mathrm{~ms} ; \tilde{\rho}=7.82 \mathrm{~ms} ; \tilde{\rho}=338-377$ ms (width: $39 \mathrm{~ms}$ ).

The most commonly-used $\mathrm{T}$ wave features are the extreme absolute value of the $\mathrm{T}$ wave (its apex_T value) and the markers taken to be signalling the completion of ventricular repolarization. The timing markers commonly used are illustrated Fig.8, on the recorded signals of leads V1 and V4, also shown in Fig.4. The marker $a$ is the timing of the apex, $b$ the timing of the inflection point of the segment following apex_T and $c$ is the timing of the intersection of the tangent at time $t=b$ with the baseline.

The statistics of the timing markers $a, b$, and $c$ observed on leads V1 and V4 of subject HM are listed in Table 1. All such markers are commonly taken as indicating the end of the 
repolarization, frequently referred to as "the end" of the $\mathrm{T}$ wave. In addition, Table 1 shows the minimum, mean and maximum values observed on the 64-leads, as well as those observed on $\operatorname{STD}(t)$, the signal that expresses the time course of the standard deviation of the lead potentials. This signal provides a robust, accurate view on the global timing of both depolarization and repolarization as recorded from any arbitrary lead system. It is a signal that is similar, but superior to, the better-known RMS(t) curve [37]. The superiority stems from the fact that the $\operatorname{STD}(t)$ signal does not depend on the potential reference involved.

Table 1. Statistics of the markers of the timing of endrepolarization $a, b$, and $c$ (specified on Figs.8a,b) as observed on leads V1, V4; the 64-lead system and $\operatorname{STD}(t)$ function. For the 64leads minimum, mean and maximum values are shown. The time $t=0$ is taken at the onset of the QRS complex.

\begin{tabular}{|c|c|c|c|}
\hline & $a(\mathrm{~ms})$ & $b(\mathrm{~ms})$ & $c(\mathrm{~ms})$ \\
\hline $\mathrm{V} 1$ & 374 & 396 & 46 \\
\hline $\mathrm{V} 4$ & 359 & 394 & 423 \\
\hline 64-leads & 316356385 & 351391420 & 367417467 \\
\hline $\mathrm{STD}(t)$ & 358 & 393 & 419 \\
\hline
\end{tabular}

The values observed on the individual signals of the 64lead system were strongly correlated, the highest value of the linear correlation coefficient being $0.96(b$ and $c)$, the lowest $0.88(a$ and $c$ ). This suggests that, apart from a shift in their mean value in a direction that is obvious from their definition (Fig.8), these markers $b$ and $c$ provide the same estimate of end-repolarization as is observable in individual signals.

In clinical data, the signal quality and that of the subsequently applied signal processing (baseline correction) is not always sufficient to make a robust estimation of markers $b$ and $c$. Frequently, their values in individual leads are ignored in reports about their potential diagnostic value. Moreover, the presence of the U wave (V1, Fig.8) tends to be ignored also, or heavily masked by assigning the end of the baseline correction to the early stage of the $U$ wave. The end of the $T$ wave, estimated by either of the markers $b$ and $c$, does not signal the end of repolarization (Calcium dynamics not having returned to any stable state) whatever their effectiveness may seemed to have been on the basis of statistical studies. Moreover, normal values like the length of the QT interval are lead-specific: any report on QT interval data should specify the lead(s) involved, the type of the baseline correction applied and the definition of "end of T" used. Without such data the documented values cannot be interpreted.

The mean timing of the apex of the T wave, marker $a$, has a special significance. A comparison with the mean value of the repolarization parameter found in the inverse procedure ( $\bar{\rho}=355 \mathrm{~ms}$ ) shows that both are equal. For the normal ECG, the mean timing of the $\mathrm{T}$ waves is the mean value of the timing of the inflection point of the down slope of the transmembrane potentials. The correspondence is in particular close if the range of the $\rho$ values is small. Moreover, in that situation the shape of a "typical" $\mathrm{T}$ wave, such as the one shown for lead V4 (Fig.7), resembles the derivatives of any of the transmembrane potential waveforms, which under these conditions have very similar shapes. They are almost replicas of one another, each approximately just being shifted and slightly stretched in time according to the values of $\delta_{n}$ and $\rho_{n}$. A mathematical analysis of this property, essentially based directly on (9), can be found in [38].

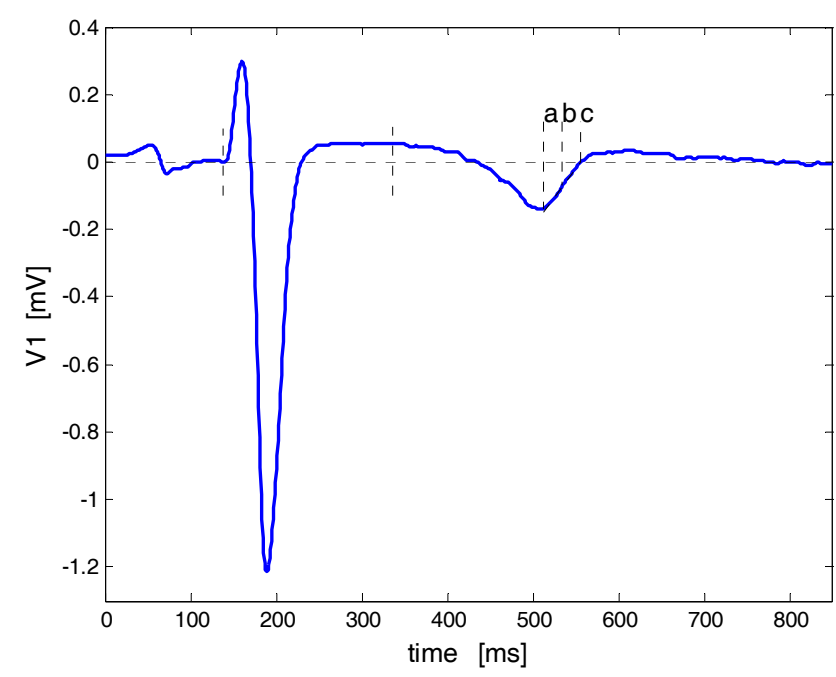

Fig.8a Markers of timing indicated on measured leads V1. From left to right: onset of QRS, mid QT interval; $a$ : the timing of the apex, b: that of the subsequent inflection point, c: the intersection of the tangent at $\mathrm{t}=\mathrm{b}$ and the baseline. Linear baseline correction was applied from data at the onset QRS to the end of the signal segment show: the beginning of the subsequent $P$ wave (not shown).

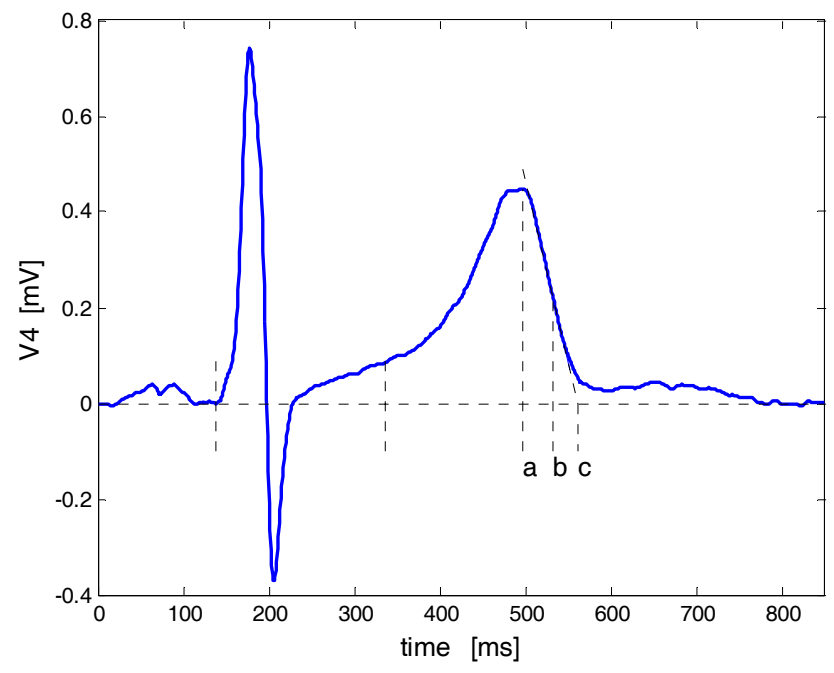

Fig.8b As in Fig.8a, now for lead V4. 


\section{$B$ Timing of Apex_T}

The significance of the amplitude of the $\mathrm{T}$ wave can also be interpreted on the basis of the EDL source model. An example of this is illustrated in Fig.9. It demonstrates the effect of the dispersion of the timing of repolarization on $\mathrm{T}$ wave magnitude as observed in simulations based on the EDL source model: Fig.9a: lead V1, Fig.9b: lead V4.

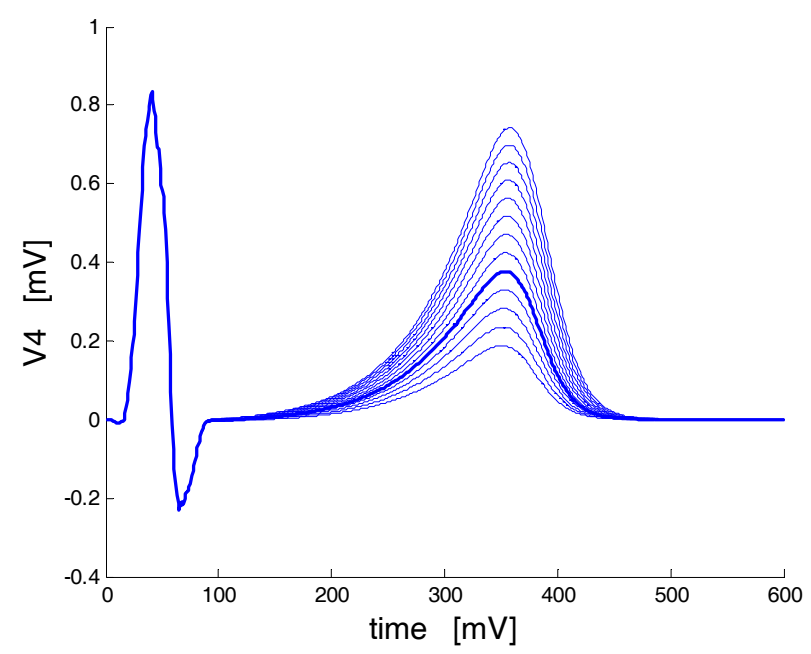

Fig.9a The effect of the dispersion of the timing of repolarization on $\mathrm{T}$ wave magnitude as observed in simulations based on the EDL source model; lead V1. With reference to the dispersion involved in the simulation most closely resembling the measured data (heavy trace), the other traces result from scaling the dispersion by factors ranging from 0.5 to 2 , in 12 steps of 0.25 , while keeping the spatial distribution (its pattern) of the $\rho$ values the same. Note that the major effect of this is a proportional scaling of $\mathrm{T}$ wave magnitude.

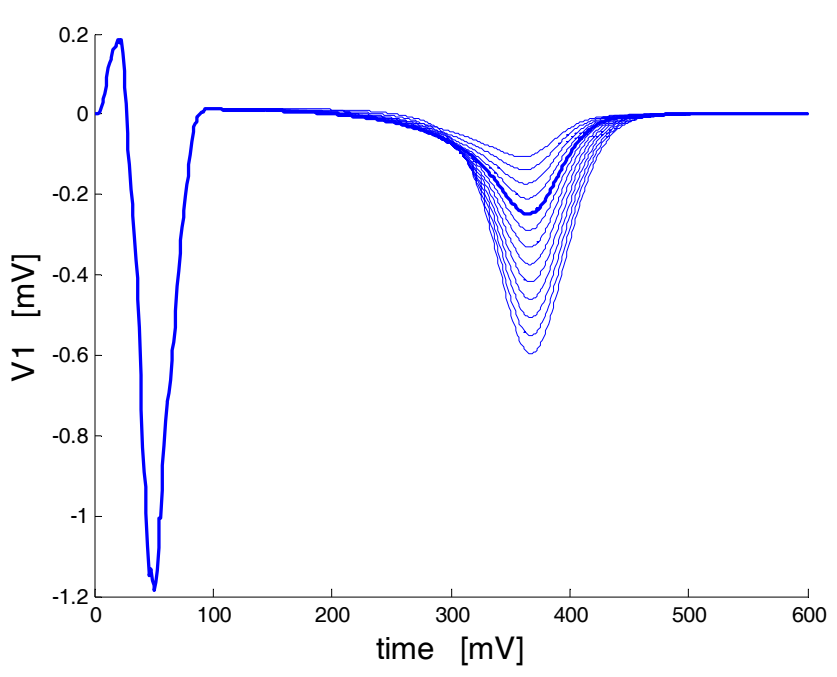

Fig.9b As in Fig.9a, now for lead V4.
With reference to the dispersion involved in the simulation most closely resembling the measured data, the other traces result from scaling the dispersion by factors ranging from 0.5 to 2 , in 12 steps of 0.25 , while keeping the spatial distribution (pattern) of the $\rho$ values the same. The effect of scaling the dispersion resulted in the proportional scaling of the $T$ wave magnitudes of all leads by a uniform (but different) factor. Significantly, when using a scaling factor of zero (not shown), associated with a hypothetical uniform (non-dispersed) timing of repolarization throughout the myocardium, the resulting magnitude of the $\mathrm{T}$ wave is zero in all leads.

Over the wide range of scaling factors shown, the effect on $\mathrm{T}$ wave magnitude is linear in all leads, with a uniform factor of proportionality. The effect on the timing of apex_T can be seen to be minimal. Although there is an increase in the width of the $\mathrm{T}$ wave for higher scaling values. However, its halfwidth increases only in a minor fashion unless extreme increases of the dispersion are involved, for which the actual shape of the TMP potentials involved begins to play a role.

\section{$C$ The ST segment}

As is mentioned in the Introduction, the signals during the interval leading up to its apex, the ST segments, have been found to contain useful diagnostic information, in particular with respect to episodes of acute ischemia that might lead to myocardial infarction (MI). In healthy subjects the majority of the 12-lead signals show relatively small values (Figs 4 and 8). During the various stages of acute ischemia, extreme changes in the ST level may be observed. The ST level is used to monitor the ongoing process. A direct interpretation of the ST level on the basis of the underlying electrophysiology is not always straightforward. A part of this problem relates to basic factors involved in the recording of bioelectric signals as discussed in Sect. 2. Below, ST changes as observed on the ECG are discussed, while incorporating these factors and analyzing their effect on the basis of the EDL source model.

As is discussed in Sect.2, bioelectric potentials observed by means of electrodes are contaminated by contact potentials at the electrode-tissue interface. The magnitudes of these potentials are much larger than those of the ECG, unknown, and may change slowly in time: the so-called baseline 'wander' or 'drift'. This problem is treated by including a highpass filter in the first stage of the amplifier. The resulting signal is referred to as an $\mathrm{AC}$-recording $(\mathrm{AC}=$ alternating current). This produces an output signal, which, when computed over a long time, has a zero mean value: the DC component of the signal is zero. If rapid perturbations of the contact potential occur, the baseline of the signal, i.e. the values in the observed signal that should be assigned a zero value, must be specified on a beat-to-beat basis. Even if the dynamic range of the input stage were wide enough to encompass the full range of sensor potentials (allowing a DC recording in spite of the presence of the contact potentials) the subseqent shift to a zero level remains to be defined.

The treatment of the problem is generally referred to as baseline correction. However, this is a somewhat misleading 
term since it assumes that the signal level that should be specified as zero is self-evident, which is in fact not the case. The problem involved is illustrated by the following example.

The ECG records express differences in the potential field generated at different locations on the thorax. This potential field is uniform over the thorax if all myocytes have the same transmembrane potential (1). This results in zero values in all ECG leads. In healthy myocardial tissue, the time instant at which this situation is most closely approximated is just before the beginning of atrial depolarization: the onset of the $\mathrm{P}$ wave. The signal of lead V3 shown on the upper trace of the left panel of Fig.10 is the (unprocessed) output of an AC-coupled amplifier. For this healthy subject and this particular beat, the onsets of the P waves were close to zero, and so only a minor shift was required to refer this signal to zero at the onset of the subsequent $\mathrm{P}$ waves.

The problem of baseline definition is more pressing for recordings taken during periods of acute ischemia. During ischemia, the TMPs of the myocytes within the ischemic region change: the resting potential decreases (tends to zero), the upstroke is reduced. The size of these changes varies during the various stages of ischemia [39].

The intercellular intra-cellular coupling between myocytes (I) in the ischemic region and myocytes $(\mathrm{H})$ in the healthy region and the differences in the TMPs of the myocytes within these regions result in an electric current flow. During the time interval between the end of the $\mathrm{T}$ wave and the onset of the QRS complex the differences in the resting potentials in these regions result in a current flow in the intracellular myocardium directed from region $I$ to region $\mathrm{H}$. In the extracellular domain the direction of the current flow is in the opposite direction, since charge is conserved. This can be expressed by an equivalent current sink in the ischemic region, which lowers the nearby extracellular potentials [39]. During the activation of the tissue surrounding the ischemic zone the potential difference between the regions is smaller and the loading effect is smaller.

In the lower traces of Fig.10 the effect of the current sink on the potential of lead V3 during the resting state is simulated by a downward DC shift of $0.2 \mathrm{mV}$. In this simulation, the contribution from the ischemic zone to the potential of lead V3 during the depolariazation phase was set to $-0.05 \mathrm{mV}$. Subsequently, during repolarization, the contribution of the sink slowly returns to the $-0.2 \mathrm{mV}$ level. The time course of the contribution of the current sink is shown by the dash-dot line. The addition of this contribution to the signal of the nonischemic situation (the upper trace) results in the type of waveform for the V3 signal that can be expected during the ischemic period (the lower solid trace on the left panel of Fig.10.). Note that between the end of the $T$ wave and the moment of depolarization reaches the ischemic-zone (R-S segment) the signal is lowered by $0.2 \mathrm{mV}$ and the remaining part by $0.05 \mathrm{~m} \mathrm{~V}$, then followed by a slow return to the (depressed) value of $-0.2 \mathrm{mV}$ at the end of the repolarizing phase.
Standard ECG recordings do not reveal the baseline depression shown in the lower solid trace in the right panel of Fig.10. The presence of the highpass filter results in a signal that has a zero mean value. A (subsequent) baseline "correction" to the onset of the $\mathrm{P}$ wave then results in the upper trace of the same figure. As a result of the current sink within the ischemic zone, the actual baseline depression shows up as an elevation of the ST-segment, merely as the consequence of the defining the zero baseline at the onset of either the $\mathrm{P}$ wave or the QRS complex. This phenomenon hinders a direct interpretation of the ECG features in terms of the underlying source-sink configurations associated with electrophysiology.

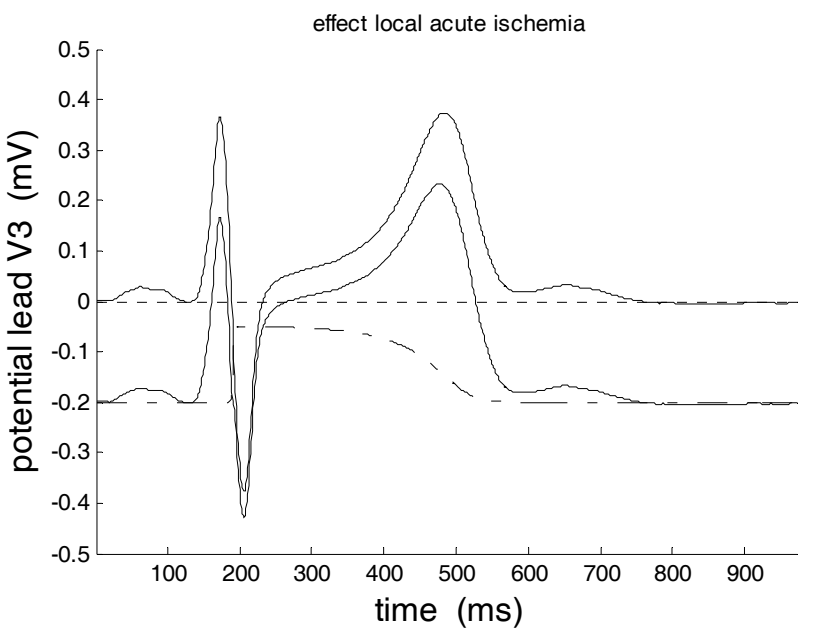

Fig.10a Lead V3 signals. Upper trace: healthy myocardium; lower trace: true, i.e. DC variant, of lead V3 during ischemia; dashed line: the difference.

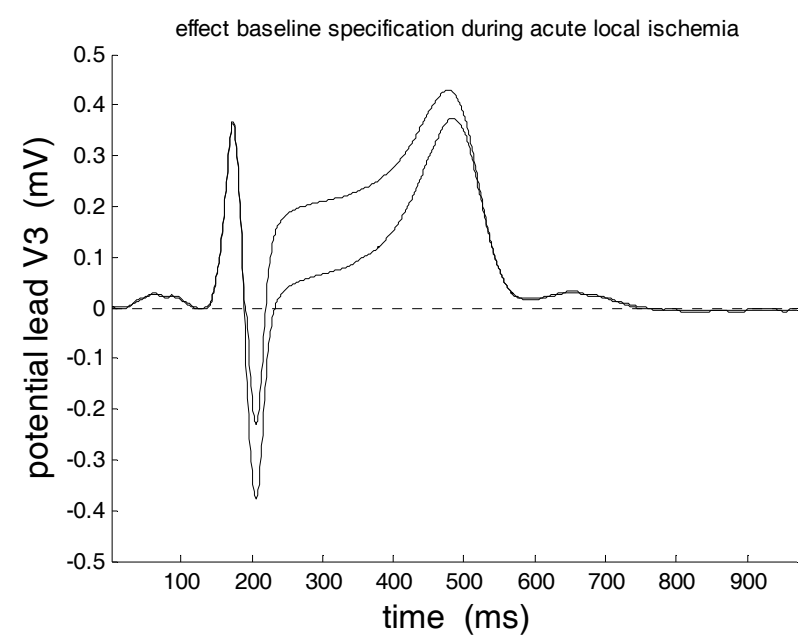

Fig.10b Lead V3 signals. Lower trace: once more the healthy situation, upper trace: the waveform as observed in the ECG after baseline "correction". Note that the true ST depression that takes place (left panel) is observed as an elevation in the ECG. 


\section{DISCUSSION}

As shown in this paper, the measurement of the $\mathrm{T}$ wave as such is a straightforward procedure. The major concern in the correct interpretation of the observed waveform features originates from the nature of the currents generating the $\mathrm{T}$ wave. During the depolarization phase, the dominant source distribution is restricted to the depolarizing wave front only. In contrast, the strength of the sources during repolarization is much weaker and their distribution is present throughout the myocardium. The lack of a complete set of invasive data on the repolarization of the entire myocardium clearly hampers the development of a validated source model. The EDL source model used in the analysis of this paper seems to be a workable model, which, like all models, may need to be replaced by an alternative should more complete data become available. The following "conclusions" are inspired by the analysis based on this model.

The analysis shows that the major significance of the timing of apex $\mathrm{T}$ is that of the mean value of the timing of the downstroke of the (mean) transmembrane potential of the myocytes. This is in fact a property that follows directly from the general expression (4), a property related to the fundamentals of volume conduction theory, and applies independently of the limiting assumptions involved in the derivation leading up to the EDL source model. This statement may seem to be in contrast to those based on measured data that state that the timing of apex-T indicates the recovery time of the epicardial surface. However, the latter statements are usually based on in vitro observations of small tissue segments. These do not take into account the full complexity of the diffuse nature of repolarization currents, which are present continuously within the entire 3D ventricular myocardium.

The results shown in Fig.9 indicated that the magnitude of the $T$ wave is an indicator of the magnitude of the dispersion of the timing of repolarization. The width of the observed $\mathrm{T}$ wave is a secondary factor, related to magnitude. In clinical situations the factor scaling the dispersion of repolarization is unknown. However, note that while monitoring ST changes in individual patients, a corresponding proportionality factor is involved, scaling all lead magnitudes in a uniform fashion. As in all other ECG wavelets, the magnitude of apex T in the individual leads of a subject depends on heart position relative to the electrode positions involved as well as on the orientation of the heart. This causes all voltage-related ECG parameters to be less robust than desirable.

The use of the EDL model in the analysis of ST changes during acute ischemia offers a solution for the current confusion about the interpretation of such observed data. It yields an explanation for the effects on body surface potentials that is consistent with observations and interpretations of results from electrophysiological studies that have been known for a long time [39]. The remaining fundamental problem is the impossibility of establishing the level of the baseline, due to the polarization effects at the electrode-tissue interfaces, which prohibits the observation of the DC shifts in the body surface potentials resulting from ischemia.

The examples shown in this paper relate to a single subject (HM) only. However, the same analysis of $\mathrm{T}$ wave data has been performed on the ECGs in a data base comprising the data of 125 subjects, leading to the same qualitative conclusions as presented here. Details of the method for finding the timing of depolarization and repolarization can be found in [29]. All examples shown can easily be replicated and studied in greater detail by using ECGSIM [31].

In conclusion, the measurement of the $\mathrm{T}$ wave and the subsequent proper interpretation of repolarization processes on the basis of observed $\mathrm{T}$ wave morphology needs to be supported by adequate modeling of the electrophysiological properties of the tissue, while also taking into account the physics involved.

\section{REFERENCES}

[1] Einthoven, W., de Lint, K. (1900). Ueber das normale menschliche Elektrokardiogram und Uber die capillarelektrometrische Untersuchung einiger Herzkranken. Pflugers Arch. ges. Physiol., 80, 139-160.

[2] Ritsema van Eck, H.J., Kors, J.A., van Herpen, G. (2005). The U wave in the electrocardiogram: a solution for a 100-year-old riddle. Cardiovasc. Res., 67, 256-262.

[3] di Bernardo, D., Murray, A. (2002). Origin on the electrocardiogram of U-waves and abnormal U-wave inversion. Cardiovasc. Res., 53, 202-208.

[4] Schalij, M.J., et al. (eds.) (2002). Einthoven 2002: 100 Yeart of Electrocardiography. Leiden, Netherlands: The Einthoven Foundation, 616.

[5] Webster, J. (ed.) (1998). Medical Instrumentation, Application and Design. 3 ed. New York: Wiley.

[6] MettingVanRijn, A.C., Kuiper, A.P., Linnenbank, A.C., Grimbergen, C.A. (1993). Patient isolation in multichannel bioelectric recordings by digital transmission through a single optical fiber. IEEE Trans. Biomed. Eng., 40 (3), 302-8.

[7] Winter, B.B., Webster, J.G. (1983). Driven-right-leg circuit design. IEEE Trans. Biomed. Eng., 30 (1), 62-66.

[8] Macfarlane, P.W. (1989). Lead systems. In Marfarlane, P.W., Lawrie, T.D.V. (eds.) Comprehensive Electrocardiology: Theory and Practice in Health and Disease. Pergamon Press, 315-352.

[9] Hoekema, R., Uijen, G.J.H., van Oosterom, A. (1999). The number of independent signals in body surface maps. Meth. Inform. Med., 38 (2), 119-124.

[10] Taccardi, B. (1963). Distribution of heart potentials on the thoracic surface of normal human subjects. Circ. Res., 12, 341-352.

[11] Macfarlane, P.W. (1989). Normal limits. In Macfarlane, P.W., Lawrie, T.T.V. (eds.) Comprehensive Electrocardiology: Theory and Practice in Health and Disease. Vol. 3. Pergamon Press, 1441-1526. 
[12] Jackman, W.M., Friday, K.J., Anderson, J.L., Aliot, E.M., Clark, M., Lazzara, R. (1988). The long QT syndromes: a critical review, new clinical observations and a unifying hypothesis. Prog. Cardiovasc. Dis., 31 (2), 115-172.

[13] Antzelevitch, C., et al. (2003). Brugada syndrome: 19922002. J. Am. Coll. Cardiol., 41 (10), 1665-1671.

[14] Gussak, I., Antzelevitch, C. (2000). Early repolarization syndrome: clinical characteristics and possible cellular and ionic mechanisms. J. Electrocardiol., 33, 299-309.

[15] Faes, T.J.C. (1992). Assessment of Cardiovascular Autonomic Function. An Inquiry into Measurement. PhD Thesis, Vrije Universiteit Amsterdam.

[16] Wilson, F.N., Macleod, A.G., Barker, P.S. (1933). The distribution of action currents produced by the heart muscle and other excitable tissues immersed in conducting media. J. Gen. Physiol., 16, 423-456.

[17] Plonsey, R. (1965). An extension of the solid angle formulation for an active cell. Biophysical J., 5, 663-666.

[18] Smythe, W.R. (1968). Static and Dynamic Electricity. New York: McGraw-Hill.

[19] Panofski, W.K.H., Phillips, M. (1962). Classical Electricity and Magnetism. London: Addison-Wesley.

[20] Geselowitz, D.B. (1989). On the theory of the electrocardiogram. Proc. IEEE, 77 (6), 857-876.

[21] van Oosterom, A. (2009). The equivalent double layer; source model for repolarization. In Macfarlane, P.W., et. al. (eds.) Comprehensive Electrocardiology. Vol. 1. New York: Springer.

[22] Geselowitz, D.B. (1992). Description of cardiac sources in anisotropic cardiac muscle. Application of bidomain model. J. Electrocardiol., 25 Suppl., 65-67.

[23] Gulrajani, R.M. (1998). Bioelectricity and Biomagnetism. New York: John Wiley \& Sons.

[24] Barr, R.C., Ramsey, M., Spach, M.S. (1977). Relating epicardial to body surface potentials by means of transfer coefficients based on geometry measurements. IEEE Trans. Biomed. Eng., 24, 1-11.

[25] Oostendorp, T.F., van Oosterom, A. (1989). Source parameter estimation in inhomogeneous volume conductors of arbitrary shape. IEEE Trans. Biomed. Eng., 36, 382-391.

[26] Huiskamp, G.J.M. (1998). Simulation of depolarization and repolarization in a membrane-equations-based model of the anisotropic ventricle. IEEE Trans. Biomed. Eng., 45 (7), 847-855.
[27] van Dam, P.M., van Oosterom, A. (2005). Volume conductor effects involved in the genesis of the $\mathrm{P}$ wave. Europace, 7, S30-S38.

[28] Huiskamp, G.J.M., van Oosterom, A. (1989). The depolarization sequence of the human heart surface computed from measured body surface potentials. IEEE Trans. Biomed. Eng., 35 (12), 1047-1058.

[29] van Dam, P.M., et al. (2009). Non-invasive imaging of cardiac activation and recovery. Ann. Biomed. Eng, (in press).

[30] Fischer, G., et al. (2005). A signal processing pipeline for noninvasive imaging of ventricular preexcitation. Meth. Inform. Med., 44, 588-515.

[31] van Oosterom, A. Oostendorp, T. (2004). ECGSIM; an interactive tool for studying the genesis of QRST waveforms. Heart, 90, 165-168.

[32] van Oosterom, A., Jacquemet, V. (2005). A parameterized description of transmembrane potentials used in forward and inverse procedures. In Int. Conf. Electrocardiol. Gdansk, Poland: Folia Cardiologica.

[33] Haws, C.W., Lux, R.L. (1990). Correlation between in vivo transmambrane action potential durations and activation-recovery intervals from electrograms. Circulation, 81 (1), 281-288.

[34] Durrer, D., et al. (1970). Total excitation of the isolated human heart. Circulation, 41, 899-912.

[35] Franz, M.R., et al. (1987). Monophasic action potential mapping in a human subject with normal electrograms: direct evidence for the genesis of the $\mathrm{T}$ wave. Circulation, 75 (2), 379-386.

[36] Cowan, J.C., et al. (1988). Sequence of epicardial repolarization and configuration of the $\mathrm{T}$ wave. $\mathrm{Br}$. Heart J., 60, 424-433.

[37] Ihara, Z., van Oosterom, A., Hoekema, R. (2006). Atrial repolarization as observable during the PQ interval. $J$. Electrocardiol., 39, 290-297.

[38] van Oosterom, A. (2002). The singular value decomposition of the $\mathrm{T}$ wave: its link with a biophysical model of repolarization. Int. J. Bioelectromagnetism, 4, 59-60.

[39] Janse, M.J., et al. (1980). Flow of injury current and patterns of excitation during early ventricular arrhythmias in acute regional myocardial ischemia in isolated porcine and canaine hearts; evidence for two different arrhythmogenic mechanisms. Circ. Res., 47, 151-165. 